\title{
Decreased Hypothalamic Functional Connectivity with Subgenual Cortex in Psychotic Major Depression
}

\author{
Keith Sudheimer*,1,2, Jennifer Keller', Rowena Gomez', Lakshika Tennakoon ${ }^{3}$, Allan Reiss', Amy Garrett', \\ Heather Kenna', Ruth O'Hara ${ }^{1,2}$ and Alan F Schatzberg' \\ 'Department of Psychiatry and Behavioral Sciences, Stanford University, Stanford, CA, USA; '2VA Palo Alto Sierra Pacific MIRECC and \\ Advanced Fellowship Program in Mental Health Research and Treatment, Palo Alto, CA, USA; ${ }^{3}$ Department of Surgery, Stanford University, \\ Stanford, CA, USA
}

\begin{abstract}
Hypothalamus communication with the rest of the brain and peripheral target tissues is critically important for many physiological and psychological functions. These functions include maintaining neuroendocrine circadian rhythms and managing affective processes. The hypothalamus maintains both direct neural connections within the brain and it also controls a variety of neuroendocrine processes that can influence target tissues throughout the body. Dysregulation of the hypothalamic pituitary adrenal axis and hyperactivity of the subgenual cortex are both frequently observed in depression. However, many details of how the hypothalamus, the hypothalamic pituitary adrenal (HPA) axis, and the subgenual cingulate interact with each other are unknown. We hypothesized that resting-state functional connectivity between the hypothalamus and the subgenual cortex would be associated with altered circadian rhythm in patients with depression and depressive symptoms. We also hypothesized that this would be most apparent in patients that have major depression with psychotic symptoms, who typically have the most robust HPA-axis dysregulation. Resting-state functional magnetic resonance imaging ( $\mathrm{fMRI}$ ) scans were collected to observe low-frequency resting-state functional connectivity patterns of the hypothalamus in 39 healthy participants, 39 patients with major depression, and 22 patients with major depression with psychotic symptoms. Hourly overnight measures of cortisol secretion and multiple measures of psychiatric symptom severity were also collected on all. Strong hypothalamic functional connectivity with the subgenual cortex was observed in healthy participants. This connectivity was significantly reduced in patients with psychotic major depression. Increased cortisol secretion during the circadian nadir and reduced connectivity were both associated with symptom severity. Reduced connectivity and high cortisol secretion during the circadian nadir are both useful for explaining a significant amount of variance in symptom severity that occurs between healthy participants and depressed patients. However, only cortisol secretion was useful for explaining the severity of symptoms within the depressed groups. This study suggests that the communication between the hypothalamus and the subgenual cortex is disrupted in patients with major depression with psychotic features. It also suggests that these disruptions are associated with increased symptom severity and may be a cause or a consequence of cortisol dysregulation.
\end{abstract}

Neuropsychopharmacology (20I5) 40, 849-860; doi:I0.1038/npp.20I4.259; published online 12 November 20।4

\section{INTRODUCTION}

Hypothalamus communication with the rest of the brain is critical for accomplishing a wide variety of physiological and psychological functions, including maintaining neuroendocrine circadian rhythms and managing affective processes. To accomplish these functions, the hypothalamus maintains both neural connections within the brain and also coordinates a variety of neuroendocrine cascades that can influence target tissue throughout the body. Little is known about how these neural and neuroendocrine func-

\footnotetext{
*Correspondence: Dr K Sudheimer, Department of Psychiatry and Behavioral Sciences, Stanford University, 40I Quarry Road, Stanford, CA 94305, USA, Tel: + I 65070 | 3674, Fax: + I 6504985294 ,

E-mail: ksudheim@stanford.edu

Received 15 May 2014; revised 22 August 2014; accepted 26 August 2014; accepted article preview online 8 October 2014
}

tions of the hypothalamus interact with each other in healthy individuals or in individuals with psychiatric diseases that are characterized by neuroendocrine dysregulation, such as major depression.

Dysregulation or hyperactivity of the hypothalamic pituitary adrenal (HPA) axis is well characterized in major depression (Hamani et al, 2011; Stetler and Miller, 2011). Through the HPA axis, the hypothalamus drives both the acute cortisol response to stress and cortisol secretion in a circadian rhythm. Several types of HPA disturbances are associated with depression, including cortisol hypersecretion (Carroll et al, 1976), reduced glucocorticoid receptor mRNA expression (Webster et al, 2002), and decreased glucocorticoid-induced inhibitory feedback to the HPA axis (Greden et al, 1983; Zobel et al, 1999; 2001). Although not all patients with major depression have HPA axis dysregulation, this finding is commonly observed across many studies of depressed patients. The depression subtype that 
shows the most consistent and robust HPA dysregulation is major depression with psychotic features (Stetler and Miller, 2011). These patients present with elevated nocturnal cortisol secretion (Keller et al, 2006) and decreased sensitivity to inhibitory HPA axis feedback mediated by both glucocorticoid receptor (Nelson and Davis, 1997) and mineralocorticoid receptor (Lembke et al, 2012).

Subgenual cortex hyperactivity is another commonly observed phenomenon in patients with depression. The subgenual cortex is highly active during the normal transient experience of sadness in healthy individuals and is hyperactive in depressed patients (Mayberg et al, 1999; Phan et al, 2002). This hyperactivity has been shown to correlate with the severity of depressive symptoms (Osuch et al, 2000) and normalizes after successful treatment via pharmacological and behavioral interventions (Mayberg et al, 1999). Deep brain stimulation has been used effectively to interrupt subgenual cortex hyperactivity, resulting in amelioration of depressive symptoms (Lozano et al, 2012). Subgenual cortex activity has also been shown to correlate with other autonomic markers associated with depression including vagal nerve activity and cardiac responses (Lane et al, 2013), suggesting that subgenual activity is involved not only in the psychological and neurological aspects of the experience of symptoms, but also the peripheral physiological manifestations of those symptoms.

The subgenual cortex is located in close proximity to the hypothalamus in both the human and non-human primate brains. Anatomical tract tracing studies in non-human primates have demonstrated that there are direct reciprocal projections between the subgenual cortex and the hypothalamus (Ongür et al, 1998a; Rempel-Clower and Barbas, 1998; Veazey et al, 1982). Diffusion tensor imaging studies in humans suggest that these same pathways are conserved in humans (Johansen-Berg et al, 2008). The existence of these anatomical pathways suggests direct neural communication between the subgenual cortex and the hypothalamus.

There is also evidence for indirect cortisol-mediated influence of the hypothalamus on the subgenual cortex. Corticosteroid receptors are expressed throughout the frontal cortex, including the subgenual cortex and hypothalamus (Patel et al, 2000). Activity in the subgenual cortex has been shown to be modulated by exogenous cortisol (Sudheimer et al, 2013). Taken together, this evidence suggests that the hypothalamus could be exerting influence over the subgenual cortex by way of direct neural connections and also indirectly via the HPA axis. As both HPA axis activity and subgenual cortex activity are closely linked with depression, we theorized that alterations in neural and/ or neuroendocrine communication between the hypothalamus and the subgenual cortex could be directly driving disease-related processes in patients with non-psychotic major depression (NPMD) and depression with psychotic symptoms (PMD).

The current study was designed to determine if communication between the hypothalamus and the subgenual cortex is disturbed in patients with NPMD or PMD. To do so, a fMRI study was designed to measure resting-state functional connectivity between the hypothalamus and subgenual cortex activity in healthy participants, patients with NPMD, and patients with PMD. Resting-state func- tional connectivity is a measure of the synchronization of discrete brain regions in low-frequency oscillations of the blood-oxygen level-dependent fMRI signal.

The main a priori hypothesis was that patients with major depression would have degraded hypothalamic resting-state functional connectivity with the subgenual cortex and that these disruptions would be associated with HPA axis dysregulation and greater severity of depressive symptoms. Patients with PMD were hypothesized to have the most degraded connectivity, the greatest cortisol dysregulation, and the most severe symptoms. In addition to resting-state fMRI, all participants also completed standard clinical scales to quantify depression severity and hourly overnight blood draws to characterize the circadian rhythm of cortisol secretion.

\section{MATERIALS AND METHODS}

\section{Participants}

A total of 113 participants (ages 18-70 years of age, Table 1) were recruited from the local community, hospitals, print advertisements, radio advertisements, and treatment clinics via referrals. All participants gave written informed consent in accordance with local Stanford University internal review board guidelines. A total of 13 of these patients were excluded from analysis due to motion artifacts, susceptibility artifacts or other artifacts that affected the quality of the neuroimaging data. The final study groups consisted of 100 participants: 39 healthy controls, 39 depressed patients without psychotic symptoms (NPMD), and 22 depressed patients with psychotic symptoms (PMD). One of the final healthy control participants was also missing overnight cortisol measures. Therefore, analyses that include cortisol samples had 38 healthy participants.

Patients were diagnosed using the Structured Clinical Interview for DSM-IV criteria. All depressed patients (NPMD and PMD) were required to have a minimum score of 18 or higher on the Hamilton Depression Ratings scale (Hamilton, 1960) and a minimum score of 6 or higher on the seven-item version of the endogenomorphic depression subscale (Thase et al, 1983).

Patients with PMD were required to meet DSM-IV criteria for major depression with psychotic features at the time of the study and have active positive symptoms. These symptoms were quantified using the positive symptoms subscale of the Brief Psychiatric Ratings Scale (BPRS; Rhoades and Overall, 1988). PMD patients were all outpatients but under close supervision from their physician. All participants were excluded if they were pregnant, lactating, using illicit drugs, or abusing alcohol during the week before the study. Healthy control participants were excluded based on any history of axis I or II psychiatric disorders. Depressed patients were also excluded if they had electroconvulsive therapy within 4 months, were suicidal, or had a history of substance abuse within 6 months of the study. For ethical reasons, all patients continued any current psychiatric medication during the study but were required to maintain a stable medication regimen for at least 1 week before the study. Amongst both depressed patient groups, there were some patients with secondary (comorbid) psychiatric disorders (see Table 2). 
Table I Means and Standard Errors (in Parentheses) for Each Group Are Listed Along with Significant Effects Observed in Omnibus/Follow-up Tests from a Multivariate ANOVA

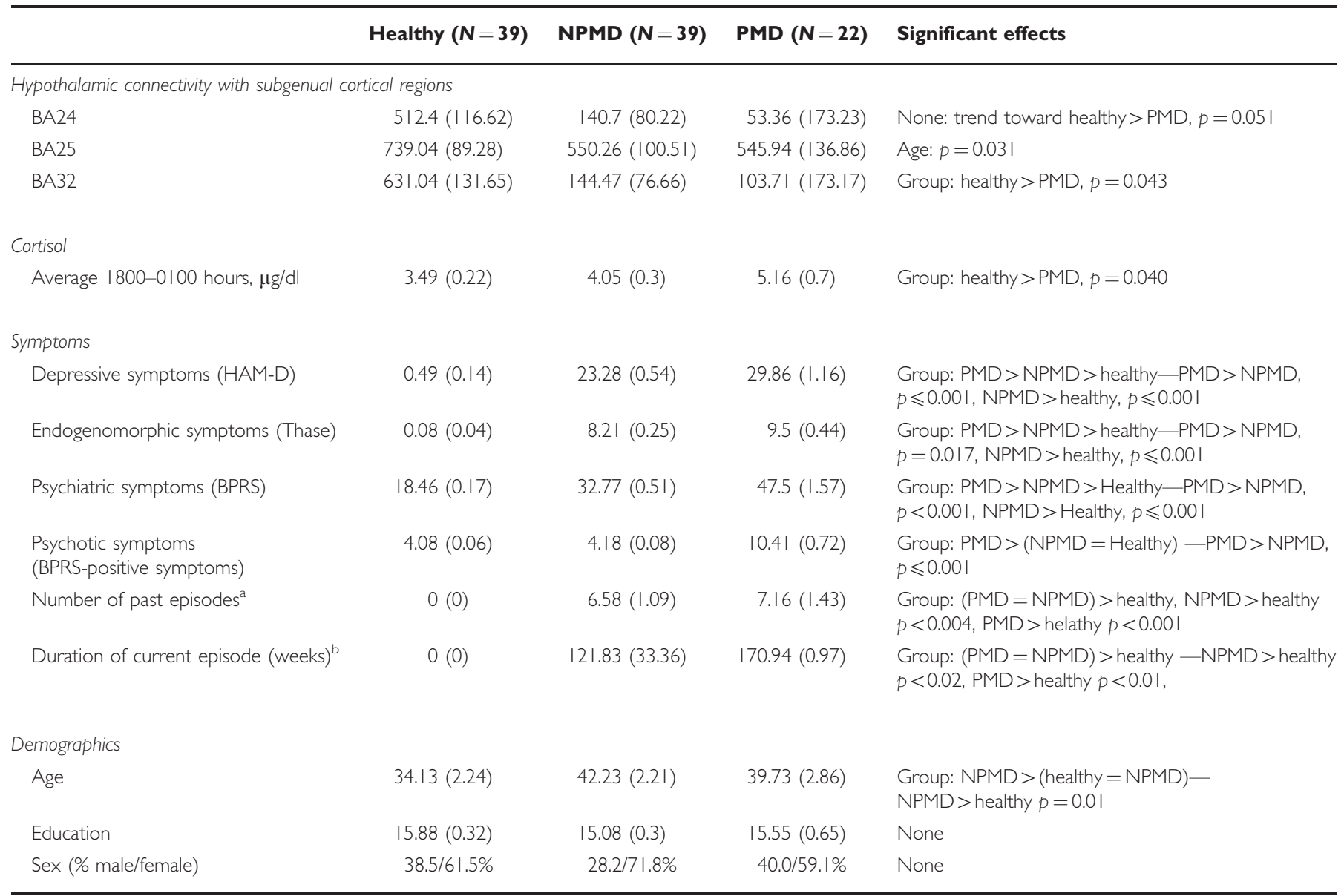

Abbreviations: NPMD, non-psychotic major depression; PMD, psychotic major depression.

${ }^{a} \operatorname{NPMD}(N=23), \operatorname{PMD}(N=19)$.

bNPMD $(N=29)$, PMD $(N=18)$.

Follow up tests were $t$-tests or $\chi^{2}$ (for gender).

\section{Protocol}

A between-groups design was used. Each participant in the three groups completed a comprehensive psychiatric assessment, hourly overnight blood draws from 1800 to 0900 hours to measure circadian cortisol secretion, and a fMRI scanning session.

fMRI volumes were collected over a 5-min scan while participants rested with their eyes closed inside a 3T GE Signa scanner (General Electric Corporation) equipped with a radio frequency head coil. These volumes were echo planar images with a 2-mm isotropic voxel dimensions. Functional images were slice-time corrected, realigned (motion corrected), co-registered, normalized to a standard template, and smoothed with a $8-\mathrm{mm}$ Gaussian kernel using SPM8. Smoothing was designed to be sensitive subgenual cortex target clusters. The functional time series was also detrended using a voxel-wise global signal correction (Macey et al, 2004), and band-pass filtered to isolate the typical functional connectivity frequency range (0.008$0.1 \mathrm{~Hz}$ ) from high and low-frequency noise.
Functional connectivity was assessed with a region of interest (ROI), seed-based approach using the statistical parametric mapping (SPM8) platform and Matlab (Mathworks, Natick, Massachusetts). A preprocessed time series was extracted from the hypothalamus, as defined in the Anatomical Automatic Labeling atlas (Tzourio-Mazoyer et al, 2002), and verified using other available online (Sudheimer et al, n.d.) and print atlases (Damasio, 1995; Mai et al, 1997). This subject-specific hypothalamus time series was then used to test for correlated time series across the whole brain using a simple regression first-level model. For each subject, a beta and a contrast image was created based on this regression. The voxel values in these contrast images indicate the strength of connectivity between the hypothalamus and each voxel in the entire brain. These first-level contrast images were then used as input into a second-level (random effects) model that tested for between-group differences in the strength of hypothalamic functional connectivity.

In addition to testing for group differences in hypothalamus connectivity across the whole brain, we had an a priori 
Table 2 The Number of Participants Who Were Taking Medications Are Listed by Type and Combination

\begin{tabular}{|c|c|c|c|}
\hline & \multicolumn{3}{|c|}{ Group } \\
\hline & $\begin{array}{l}\text { Healthy } \\
(N=39)\end{array}$ & $\begin{array}{c}\text { NPMD } \\
(N=39)\end{array}$ & $\begin{array}{c}\text { PMD } \\
(N=22)\end{array}$ \\
\hline \multicolumn{4}{|l|}{ Medication classes and combinations } \\
\hline No medications & 39 & 16 & 3 \\
\hline Antidepressant only & 0 & 19 & 3 \\
\hline Antipsychotic only & 0 & 0 & 0 \\
\hline Axiolytic only & 0 & 0 & 0 \\
\hline Antidepressant + antipsychotic & 0 & 0 & 7 \\
\hline Antidepressant + axiolytic & 0 & 4 & I \\
\hline Antipsychotic + axiolytic & 0 & 0 & 1 \\
\hline Antidepressant + antipsychotic + axiolytic & 0 & 0 & 7 \\
\hline \multicolumn{4}{|l|}{ Psychiatric diagnoses and co-mobidities } \\
\hline Bipolar & 0 & I & 2 \\
\hline Major depression & 0 & 39 & 22 \\
\hline Psychotic major depression & 0 & 0 & 22 \\
\hline Schizophrenia & 0 & 0 & 0 \\
\hline Schizoaffective & 0 & 0 & 0 \\
\hline Panic disorder & 0 & । & 3 \\
\hline Agoraphobia & 0 & 2 & 2 \\
\hline Social phobia & 0 & 3 & 5 \\
\hline Specific phobia & 0 & 5 & 2 \\
\hline Obsessive compulsive & 0 & 0 & 4 \\
\hline Post-traumatic stress & 0 & 8 & 4 \\
\hline Generalized anxiety disorder & 0 & 3 & 3 \\
\hline
\end{tabular}

Abbreviations: NMPD, non-psychotic major depression; PMD, psychotic major depression.

Some patients present with multiple diagnoses.

hypothesis of disrupted connectivity between the hypothalamus and the subgenual cortex. However, the exact regions of the subgenual cortex that have been implicated in depression are somewhat variable. Across a number of studies the regions implicated generally conform to parts of (but not the entire extent of) Brodmann areas (BA) 25, 24, 32 and the subcallosal gyrus (Hamani et al, 2011). Therefore, smoothed binary masks were created from these regions using the spatial definitions provided by the Automatic Anatomical Labeling atlas (Tzourio-Mazoyer et al, 2002). However, as BA24 and BA32 extend dorsally beyond the subgenual regions that are generally implicated in major depression, the masks were restricted to midline frontal regions that are ventral to the genu of the corpus callosum ( $x=-20$ to $+20, y=0$ to $+50, z<0)$ (Figure 1). These ROIs were used in small volume corrections (SVC), as a compliment to the whole-brain hypothalamus connectivity analysis. In order for a SVC, a cluster of voxels was considered to be significant if it met an initial uncorrected threshold of $p<0.001$ and a cluster threshold of 10 voxels, and then also met a family-wise corrected threshold of $p<0.05$ within any of the small volumes. SVC FWE corrections were made within each subregion.

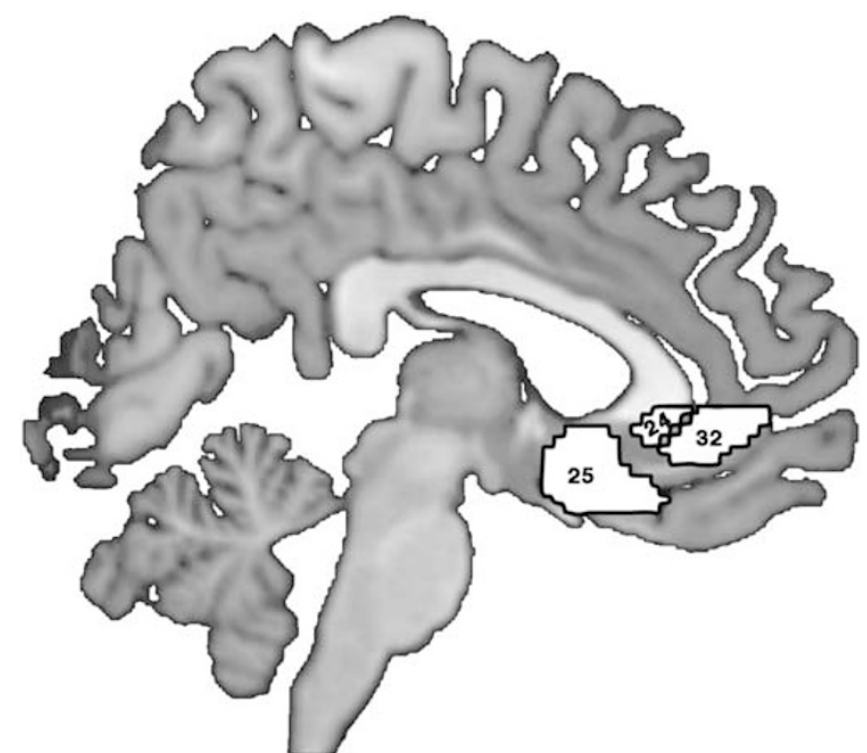

Figure I Anatomically defined regions of interest (ROI) were used for Broadmann areas (BA) 24, BA25, and BA32. The ROls were smoothed to match to spatial resolution of the functional connectivity data and then converted to binary mask images. The spatial extent was also limited to reflect the typical boundaries of subgenual cortex (ie, limited to cortex inferior to the genu of the corpus callosum).

Measures of the circadian rhythm of cortisol secretions were collected overnight at hourly intervals from 1800 to 0800 hours, for a total of 15 blood samples. Some of these data were reported in previous publications. Blood ( $8 \mathrm{ml} / \mathrm{sample})$ was drawn from an indwelling venous catheter. Plasma was immediately separated from whole blood by centrifugation and then stored at $-70{ }^{\circ} \mathrm{C}$ until assays were performed. Plasma cortisol concentrations were measured using the Access Immunoassay System (Beckman Coulter, Chaska, MN). The sensitivity of this assay is $0.4 \mathrm{mcg} / \mathrm{dl}$ $(11 \mathrm{mmol} / \mathrm{l})$ and the precision within assays is $6.4-7.9 \%$. Cortisol was also measured in the saliva of a subsample of 30 healthy, 36 NPMD, and 16 PMD patients using the Salivette cotton swab system (Sarstedt, Nümbrecht, Germany) and was analyzed by Salimetrics (State College, PA) using an enzyme immunoassay with a lower limit sensitivity of $0.003 \mu \mathrm{g} / \mathrm{dl}$ and average intra-assay and inter-assay coefficients of variation of 3.5 and $5.1 \%$. Two samples were collected from each participant, one before, and one after the fMRI scan. The mean of these two measures was used to estimate salivary cortisol during scanning.

\section{Data Analysis}

Cortisol. To determine group differences in plasma cortisol, a repeated-measures ANOVA with linearly interpolated missing values was conducted. Since there was also an a priori hypotheses regarding elevated cortisol during the circadian nadir, these values were also averaged across the 1800 to 0100 hours time window, which constitutes the typical circadian nadir. These averages were compared across groups using a standard ANOVA. Salivary cortisol measures collected on a subset of participants were averaged across 
the samples collected immediately before and immediately after fMRI scanning these values were also analyzed using a standard ANOVA model.

Functional connectivity. In addition to whole-brain and SVC analyses using SPM, we also conducted a multivariate ANOVA (MANOVA) using extracted average functional connectivity from our subgenual ROIs, plasma cortisol measures, and measures of symptom severity. This MANOVA controlled for both age and gender as factors while testing for group differences in functional connectivity, cortisol secretion, and symptom severity.

Relationships between hypothalamic functional connectivity, cortisol, and symptoms. Backward-stepwise multivariate linear regressions were conducted using SPSS to test for the relative contributions of age, gender, cortisol secretion, and functional connectivity measure to depressive symptoms. As variance across all groups and variance within the depressed group are both potentially informative these regressions were conducted across the whole sample, and a separate regression was also conducted just using all depressed patients (NPMD and PMD). Similar regression analyses were conducted to test the relative contributions of age, gender, cortisol secretion, and symptoms to functional connectivity disturbances.

\section{RESULTS}

All three participant groups demonstrated positive connectivity between the hypothalamus seed region and voxels located within or adjacent to the hypothalamus. Healthy participants exhibited strong hypothalamic connectivity to the subgenual cortex, insula, hippocampus, and superior/ middle temporal gyri (Table 3, Figure 2). This pattern of strong hypothalamic connectivity was reduced in the NPMD and PMD patients (Figure 2). Formal statistical group comparisons indicated clusters of voxels in which the strength of the hypothalamic connectivity to the subgenual cortex was significantly lower in both NPMD and PMD patients compared with the healthy control group (Figure 3). These reductions in connectivity in patients were significant at a FWE threshold of $p<0.05$ after SVC when comparing either group of patients to healthy participants (Table 3 ). No significant differences in hypothalamic connectivity were seen between the NPMD and PMD groups.

In addition to the whole-brain analysis, the strength of the hypothalamic functional connectivity was also averaged across each of the three ROI. These ROI-averaged values were corrected for age and gender inside a MANOVA model. Across all three ROIs hypothalamic connectivity to subgenual cortex showed a stepwise reduction when comparing across groups with healthy participants having the highest connectivity and PMD patients having the lowest (Figure 4). However, there was only a significant omnibus effect of group for the subgenual BA32 region. This effect was driven by lower connectivity in the PMD group $v s$ the healthy group (Table 2). In BA24, the omnibus effect of group on hypothalamic connectivity was just subthreshold for significance at $p=0.051$, which was driven by a trend toward reduced connectivity in the PMD patients relative to the healthy participants.

A significant effect of age on hypothalamic connectivity to BA25 was also observed. This effect was driven by reduced connectivity in older participants relative to younger participants (Table 2).

Cortisol secretions during the circadian nadir hours of 1800 to 0100 hours were significantly elevated in PMD patients. However, these differences did not persist throughout the whole circadian curve or into the early morning rising phase (Table 1, Figure 5). There were also no group differences observed in the sample of participants that had salivary cortisol collected at the time of scanning.

Across all participants, more severe symptoms as measured by the HAM-D total score, the endogenomorphic subscale, or the BPRS, were associated with both decreased hypothalamic connectivity to the subgenual BA32 and increased cortisol secretion during the circadian nadir. When these same analyses were conducted across the subset of only depressed patients (NPMD and PMD), only cortisol secretion was associated with increased symptoms on the HAM-D and endogenomorphic subscale (Table 4).

Across all participants, more severe disruptions in hypothalamic connectivity were negatively correlated with cortisol secretion at a trend-level significance (BA24 $r=-0.170, p=0.092$; BA25 $r=-0.179, p=0.077$; BA32 $r=-0.196, p=0.052)$. However, these effects were not significant after correcting for age, gender, or symptoms in a regression analysis. Similar non-significant negative correlations were also seen when the analysis was conducted across the subset of only depressed patients.

\section{DISCUSSION}

This study is the first whole-brain characterization of resting-state functional connectivity patterns of the hypothalamus. The findings suggest that in healthy participants there is strong hypothalamic connectivity with the subgenual cortex and other regions including the insula, superior, and middle temporal gyri. The existence of a neural circuit that could be driving these functional connectivity relationships is strongly supported by tract tracing studies in non-human primates (Johansen-Berg et al, 2008; Ongür et al, 1998a) and diffusion tensor imaging studies in humans (Johansen-Berg et al, 2008; Ongür et al, 1998b).

These data also provide the first evidence that hypothalamic connectivity with the subgenual cortex is disrupted in patients with PMD, suggesting that aspects of the pathophysiology of depression could be associated with these changes. Although only the PMD patients presented with statistically significant reduced functional connectivity, it is noteworthy that across each of the subgenual ROIs, a stepwise reduction in hypothalamic connectivity was observed with healthy controls having the strongest connectivity, followed by the NPMD patients, and PMD patients having the weakest connectivity (Figure 4).

Multiple regression models (Table 4) suggest that the large symptom differences that occur between healthy individuals and depressed individuals could be related to both excessive cortisol secretion during the circadian nadir and degraded functional connectivity between the 
Table 3 Whole-Brain and Small Volume Corrected Neuroimaging Results Show Robust Hypothalamic Connectivity with the Subgenual Cortex, the Insula, Temporal Lobe, and Several Other Subcortical Regions in Healthy Participants

\begin{tabular}{|c|c|c|c|c|c|c|c|}
\hline \multicolumn{8}{|c|}{ Group hypothalamus functional connectivity } \\
\hline Group & Region/s & $\begin{array}{l}\text { Peak voxel } \\
(x, y, z)^{a}\end{array}$ & Cluster $^{\mathrm{b}}$ & & $\begin{array}{l}\text { P-value } \\
\text { (unc.) }^{c}\end{array}$ & $\begin{array}{c}\text { P-value } \\
\text { (FWE-SVC) }^{\text {d }}\end{array}$ & $\begin{array}{l}\text { P-value } \\
\text { (FWE-Whole } \\
\text { Brain) }\end{array}$ \\
\hline \multicolumn{8}{|c|}{ Healthy $>0$} \\
\hline & $\begin{array}{l}\text { Anterior cingulate, subgenual cortex, subcallosal gyrus, rectal gyrus, } \\
\text { parahippocampal gyrus, lingual gyrus, orbital gyrus, inferior frontal gyrus, } \\
\text { middle frontal gyrus, superior frontal gyrus, medial frontal gyrus, culmen, } \\
\text { lentiform nucleus, thalamus, caudate, hippocampus, amygdala, } \\
\text { BAI0/I I/I3/I9/24/25/27/28/30/32/34/35/36/37/38/47, mammillary } \\
\text { body, globus pallidus, putamen, hypothalamus }\end{array}$ & $2,0,-10$ & 9903 & $\operatorname{lnf}$ & $<0.000001$ & $<0.001$ & $<0.001$ \\
\hline & Insula, middle temporal gyrus, superior temporal gyrus, BA |3/21/22/38 & $48,-6,-14$ & 202 & 5.01 & $<0.000001$ & - & 0.013 \\
\hline & Cerebellum: declive of vermis, nodule, pyramis, culmen & $-2,-62,-20$ & 117 & 4.15 & 0.000017 & - & - \\
\hline & Cingulate gyrus, precuneus, BA3। & $20,-54,26$ & 130 & 3.80 & 0.000073 & - & - \\
\hline & Insula, superior temporal gyrus, BA/3/22 & $-42,0,-2$ & 36 & 3.57 & $0.000|8|$ & - & - \\
\hline & Insula, precentral gyrus & $46,0,16$ & 15 & 3.50 & 0.000235 & - & - \\
\hline & Frontal lobe white matter & $26,18,36$ & 21 & 3.39 & 0.000352 & - & - \\
\hline & Superior temporal gyrus & $42,-52,20$ & 17 & 3.37 & 0.000370 & - & - \\
\hline & Temporal lobe white matter & $-26,-60,22$ & 11 & 3.22 & 0.000643 & - & - \\
\hline \multicolumn{8}{|c|}{$N P M D>0$} \\
\hline & $\begin{array}{l}\text { Anterior cingulate, subgenual cortex, subcallosal gyrus, rectal gyrus, } \\
\text { parahippocampal gyrus, orbital gyrus, superior temporal gyrus, inferior } \\
\text { frontal gyrus, middle frontal gyrus, superior frontal gyrus, medial frontal } \\
\text { gyrus, lentiform nucleus, caudate, amygdala, ba I } 0 / 11 / 13 / 24 / 25 / 28 / 32 / 34 / 38 / 47 \text {, } \\
\text { globus pallidus, putamen, hypothalamus }\end{array}$ & $2,0,-12$ & 4852 & 7.67 & $<0.000001$ & $<0.001$ & $<0.001$ \\
\hline & Middle occipital gyrus & $-34,-72,0$ & 108 & 3.99 & 0.000034 & - & - \\
\hline & Middle temporal gyrus, superior temporal gyrus, BA39 & $-30,-68,20$ & 115 & 3.93 & 0.000042 & - & - \\
\hline & Superior temporal gyrus, BA20/36/38 & $30,10,-50$ & 81 & 3.81 & 0.000069 & - & - \\
\hline & Fusiform gyrus, parahippocampal gyrus, culmen, BA20/36 & $-30,-34,-24$ & 27 & 3.60 & 0.000161 & - & - \\
\hline & Middle temporal gyrus & $-66,-70,2$ & 29 & 3.59 & 0.000165 & - & - \\
\hline & Parahippocampal gyrus, BAI9 & $-22,-44,-4$ & 39 & 3.40 & 0.000332 & - & - \\
\hline & Thalamus, pulvinar & $-16,-36,8$ & 14 & 3.31 & 0.000470 & - & - \\
\hline \multicolumn{8}{|l|}{$P M D>0$} \\
\hline & $\begin{array}{l}\text { Midbrain, anterior cingulate, subcallosal gyrus, uncus, rectal gyrus, parahippocampal } \\
\text { gyrus, inferior frontal gyrus, medial frontal gyrus, lentiform nucleus, thalamus, bal I/25/34/47, } \\
\text { medial dorsal nucleus, ventral lateral nucleus, anterior nucleus, mammillary body, } \\
\text { medial globus pallidus, putamen, hypothalamus }\end{array}$ & $0,0,-12$ & 1773 & $\operatorname{lnf}$ & $<0.000001$ & 0.008 & $<0.001$ \\
\hline & Cerebellar tonsil, pons & $18,-42,-44$ & 11 & 3.35 & 0.000400597 & - & - \\
\hline & Lentiform nucleus, thalamus, putamen & $26,-10,8$ & 13 & 3.18 & 0.000724495 & - & - \\
\hline
\end{tabular}


Table 3 (Continued)

Group hypothalamus functional connectivity

\begin{tabular}{|c|c|c|c|c|c|c|}
\hline Group & Region/s & $\begin{array}{l}\text { Peak voxel } \\
\quad(x, y, z)^{a}\end{array}$ & Cluster $^{b}$ & $\begin{array}{l}\text { P-value } \\
\text { (unc.) }^{c}\end{array}$ & $\begin{array}{c}\text { P-value } \\
(\text { FWE-SVC) }\end{array}$ & $\begin{array}{c}\text { P-value } \\
\text { (FWE-Whole } \\
\text { Brain) }\end{array}$ \\
\hline
\end{tabular}

Group differences in hypothalamus functional connectivity

Group comparison

Healthy $>P M D$

Precuneus, BA7

Precuneus

Superior frontal gyrus, medial frontal gyrus, BAIO

Parahippocampal gyrus, BAI9

Medial frontal gyrus

Subgenual cortex, anterior cingulate, medial frontal gyrus, BAI0/I I/32

Precuneus, BA7

Medial frontal gyrus

Cingulate gyrus, BA3

$\begin{array}{cccc}16,-64,42 & 34 & 3.72 & 0.000098 \\ -26,-60,22 & 50 & 3.65 & 0.000131 \\ 10,62,-6 & 33 & 3.50 & 0.000236 \\ -36,-52,-4 & 42 & 3.46 & 0.000274 \\ 36,46,-2 & 18 & 3.45 & 0.000285 \\ 2,44,-12 & 172 & 3.42 & 0.000314 \\ -10,-58,56 & 14 & 3.31 & 0.00046 \mid \\ -14,40,-8 & 14 & 3.28 & 0.000520 \\ 18,-40,30 & 12 & 3.24 & 0.000592\end{array}$

PMD > healthy

Cuneus, BAI 8

Lingual gyrus, cuneus, BAI7//8

Thalamus, anterior nucleus, medial dorsal nucleus

Middle frontal gyrus, inferior frontal gyrus, BA9/46

Lingual gyrus, BAI7/18

$\begin{array}{cccc}0,-102,10 & 80 & 3.79 & 0.000075 \\ 14,-104,-10 & 189 & 3.7 \mid & 0.000104 \\ -2,-6,12 & 93 & 3.58 & 0.000174 \\ -44,16,28 & 63 & 3.57 & 0.000178 \\ -6,-104,-10 & 34 & 3.31 & 0.000462\end{array}$

Healthy $>$ NPMD

Superior temporal gyrus, middle temporal gyrus, BAI3/21/22/38

$\begin{array}{cccc}48,-6,-14 & 130 & 4.54 & 0.000003 \\ 42,18,12 & 98 & 3.91 & 0.000047 \\ -12,-6,56 & 24 & 3.72 & 0.000099 \\ 16,-6,66 & 38 & 3.72 & 0.000101 \\ -44,0,-2 & 112 & 3.70 & 0.000106 \\ 4,46,-12 & 99 & 3.58 & 0.000174 \\ 46,-2,6 & 62 & 3.51 & 0.000222 \\ 20,6,52 & 20 & 3.46 & 0.000269 \\ -34,36,4 & 10 & 3.44 & 0.000295 \\ 0,-12,-4 & 17 & 3.38 & 0.000368 \\ 34,-4,-2 & 22 & 3.36 & 0.000385 \\ -8,14,72 & 17 & 3.35 & 0.000404\end{array}$

Insula, inferior frontal gyrus, BAI3/44/45

Medial frontal gyrus, BA6

Middle frontal gyrus, superior frontal gyrus, BA6

Superior temporal gyrus, BA6//3/22

Subgenual cortex, anterior cingulate, medial frontal gyrus, BA I 0/I I/24/32

Superior temporal gyrus, precentral gyrus, BA6//3/22/44

Cingulate gyrus, superior frontal gyrus, medial frontal gyrus, BA6/32

Inferior frontal gyrus

Midbrain, thalamus, hypothalamus

Insula, claustrum

Superior frontal gyrus

$-8,14,72$

0.000404 
Table 3 (Continued)

\begin{tabular}{|c|c|c|c|c|c|c|c|}
\hline \multicolumn{8}{|c|}{ Group hypothalamus functional connectivity } \\
\hline Group & Region/s & $\begin{array}{l}\text { Peak voxel } \\
(x, y, z)^{a}\end{array}$ & Cluster $^{\mathbf{b}}$ & & $\begin{array}{l}\text { P-value } \\
\text { (unc.) }^{c}\end{array}$ & $\begin{array}{c}P \text {-value } \\
(\text { FWE-SVC) }\end{array}$ & $\begin{array}{c}\text { P-value } \\
\text { (FWE-Whole } \\
\text { Brain) }\end{array}$ \\
\hline & Cingulate gyrus & $-8,-8,32$ & 11 & 3.32 & 0.000443 & - & - \\
\hline & Insula, inferior frontal gyrus, BA I3/47 & $-36,14,-8$ & 14 & 3.30 & 0.000489 & - & - \\
\hline \multicolumn{8}{|c|}{ NPMD > healthy } \\
\hline & Cuneus, precuneus, BA7/|9/31 & $-28,-74,24$ & 58 & 3.60 & 0.000162 & - & - \\
\hline & Middle occipital gyrus, BAI9 & $44,-74,-14$ & 12 & 3.28 & 0.000523 & - & - \\
\hline \multicolumn{8}{|c|}{$N P M D>P M D$} \\
\hline & Parahippocampal gyrus, BAI9/37 & $-34,-50,-8$ & 88 & 3.71 & 0.000104 & - & - \\
\hline & Parahippocampal gyrus, BA36 & $-32,-30,-24$ & 18 & 3.62 & $0.000 \mid 46$ & - & - \\
\hline & Temporal/parietal/occipital junction & $-28,-64,24$ & 23 & 3.28 & 0.000520 & - & - \\
\hline \multicolumn{8}{|c|}{$P M D>N P M D$} \\
\hline & Lentiform nucleus, lateral globus pallidus, putamen & $-22,-8,4$ & 40 & 3.50 & 0.000228 & - & - \\
\hline & Posterior cingulate, BA29 & $2,-48,8$ & 48 & 3.45 & 0.000280 & - & - \\
\hline & Superior frontal gyrus, BAIO & $-30,70,4$ & 24 & 3.45 & 0.000283 & - & - \\
\hline & Thalamus & $-4,-6,8$ & 21 & 3.44 & 0.000295 & - & - \\
\hline & Cuneus & $4,-102,12$ & 15 & 3.34 & 0.000413 & - & - \\
\hline & Inferior frontal gyrus, BA45/46 & $-48,26,20$ & 15 & 3.34 & 0.000415 & - & - \\
\hline & Inferior frontal gyrus, BA47 & $-48,34,-12$ & 15 & 3.24 & 0.000608 & - & - \\
\hline
\end{tabular}

Abbreviations: FWE, family-wise error; NMPD, non-psychotic major depression; PMD, psychotic major depression; SVC, small volume correction.

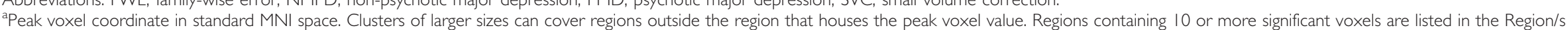
column.

${ }^{\mathrm{b}}$ Cluster size threshold was set to 10 voxels.

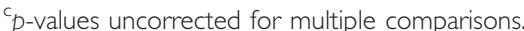

${ }^{\mathrm{d}}$ Family-wise error correction was applied within the a priori regions of interest as a small volume correction (SVC).

eFamily-wise error correction was applied.

Voxel-wise group comparisons indicate lower hypothalamic connectivity to the subgenual cortex in the depressed patients. 


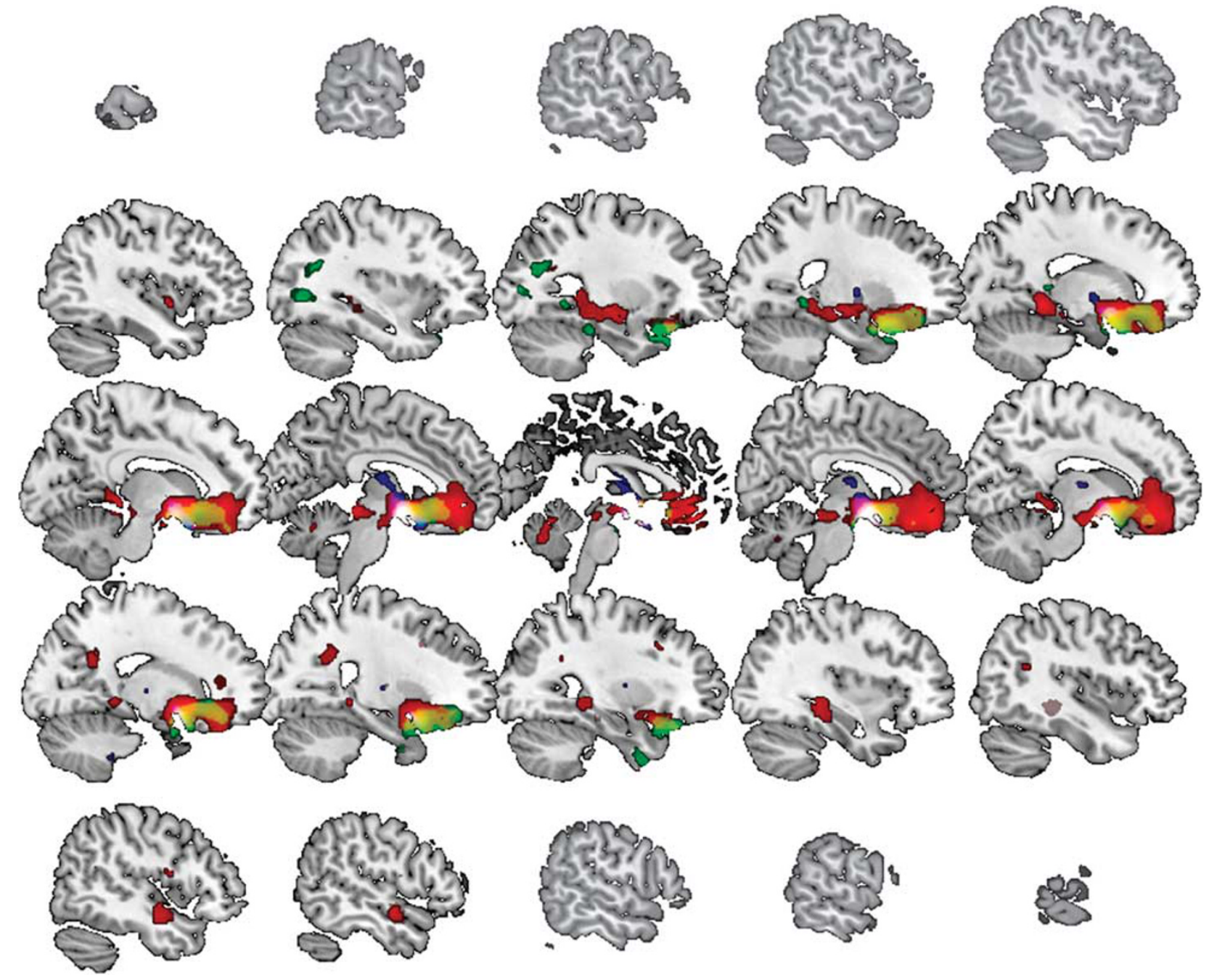

Figure 2 Healthy group, red; the non-psychotic major depression group (NPMD; green); psychotic major depression group, blue; healthy and NPMD overlap, yellow; healthy and psychotic major depression overlap, violet; NPMD and psychotic major depression overlap, cyan; and all three groups overlap, white. Conjunction maps demonstrate shared and differential spatial patterns of hypothalamic connectivity. The healthy group demonstrated significant hypothalamic connectivity to the subgenual cortex, insula, middle, and superior temporal gyri. The healthy group and the NPMD group showed some common connectivity patterns but a reduced spatial extent of hypothalamic connectivity with the subgenual cortex. The spatial extent of this connectivity is further reduced in the psychotic major depression group. All functional maps are subject to a $p<0.00$ I (unc.) voxel threshold and a cluster threshold of $k=10$ for display purposes (see Table 2 for SVC, FWE-corrected statistics).

hypothalamus and BA32. However, these models also suggest that degradations in the hypothalamic functional connectivity patterns do not explain why some NPMD or PMD patients experience more severe symptoms than others. Variance between depressed individuals on cortisol secretion was more associated with these smaller changes in symptomatology.

Lower hypothalamic connectivity with increased age is particularly notable (BA25, Table 2) as older adults generally have worse regulation of the HPA axis, and higher rates of cortisol secretion across the circadian rhythm. In our sample, cortisol secretion and age were fairly co-linear, which reduced our ability to discern the effects of age from the effects of cortisol secretion on hypothalamic connectivity to the subgenual cortex. When age is not included as a covariate in the multivariate models, significantly lower hypothalamic connectivity to BA25 was seen in the patient groups. However, the data that we present cannot resolve if age, cortisol, or both could be the main contributing factor to lower hypothalamic connectivity with BA25. Future studies with a narrower range of ages or 1:1 subject matching could resolve this ambiguity.

Previous studies have closely linked hyperactivation of the subgenual cortex with depression (Mayberg et al, 1999) and have shown that this hyperactivation normalizes with successful treatment (Mayberg et al, 1997). Prior work has also reported increased subgenual cortex connectivity with the default mode network (Grecius et al, 2007), suggesting that ramped-up subgenual activity could being a driving force behind the disruptions in hypothalamic connectivity that we observe. Glucocorticoid receptors are expressed throughout the frontal cortex in humans (Watzka et al, 2000), and cortisol can affect the emotion-related activation patterns of the subgenual cortex (Sudheimer et al, 2013). 

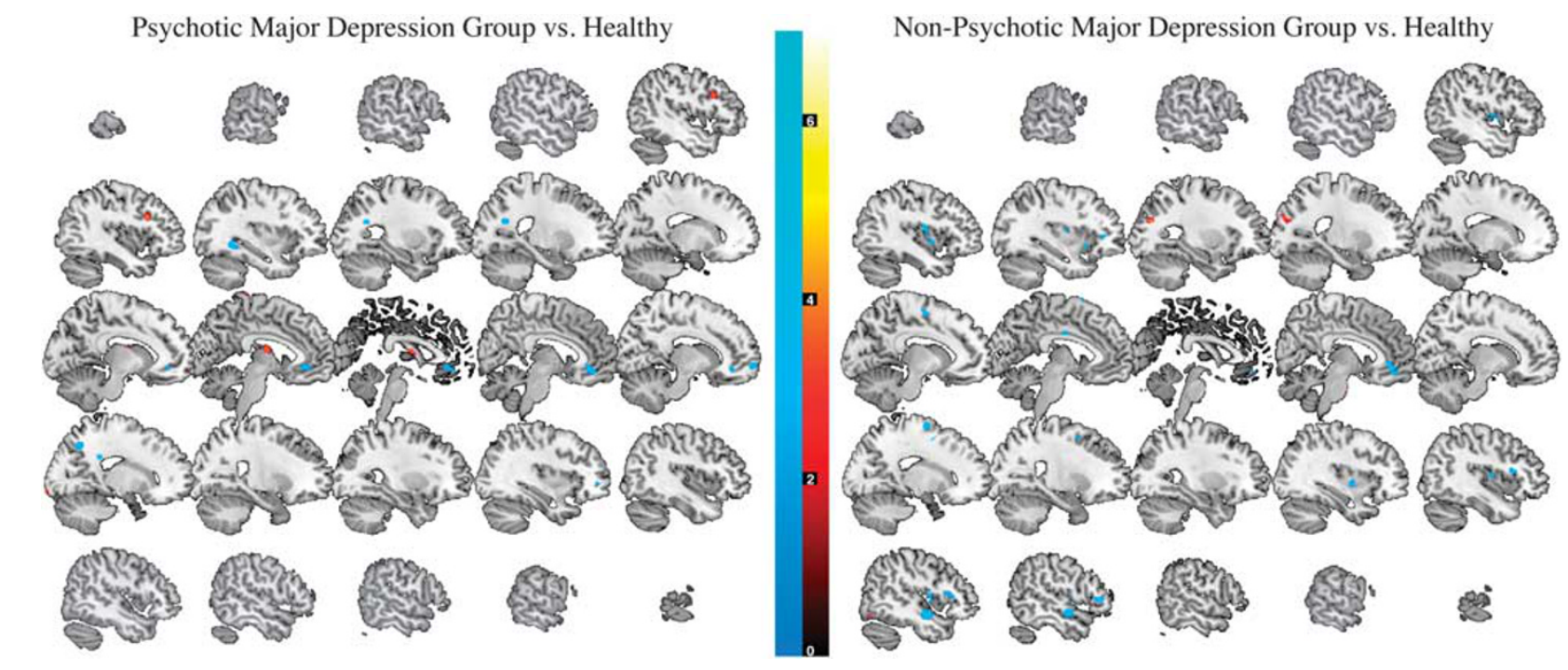

Figure 3 Whole-brain group comparison maps indicate that hypothalamic functional connectivity to the subgenual cortex is decreased in both the psychotic major depression and non-psychotic major depressiongroups (3rd row, 4th column in both maps). Functional maps are subject to a $p<0.00$ I (unc.) voxel threshold and a cluster threshold of $k=10$ for display purposes (see Table 2 for SVC, FWE-corrected statistics, and comparisons between psychotic and non-psychotic depression groups).

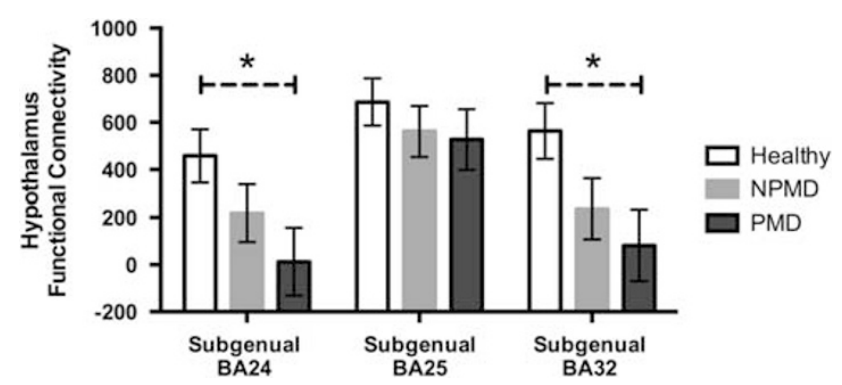

Figure 4 Age/gender corrected region of interest extractions represent connectivity between the hypothalamus seed and each of the subgenual regions averaged across all voxels. Hypothalamus connectivity is decreased in both subgenual regions BA24 and BA32, but not BA25.

These findings when taken together suggest a model of how subgenual cortex, the hypothalamus, and cortisol signaling could be interacting and affecting each other's functioning. The hypothalamus can exert control over the subgenual cortex via direct neural projections as well as indirectly through HPA-axis activation and subsequent cortisol modulation of the subgenual cortex. The subgenual cortex could also exert influence on hypothalamic and HPA axis activity via projections to the paraventricular nucleus of the hypothalamus, which is responsible for initiating corticotropin-releasing-hormone release (Rempel-Clower and Barbas, 1998). Finally, cortisol via signaling at its receptors is able to modulate both hypothalamic and subgenual cortex activity through receptors expressed in those regions.

Thus, there are many potential routes to disrupted functional connectivity between the hypothalamus and the subgenual cortex. Cortisol is capable of inhibiting both hypothalamic output and subgenual cingulate functions, but could be doing so with differential efficacy based on

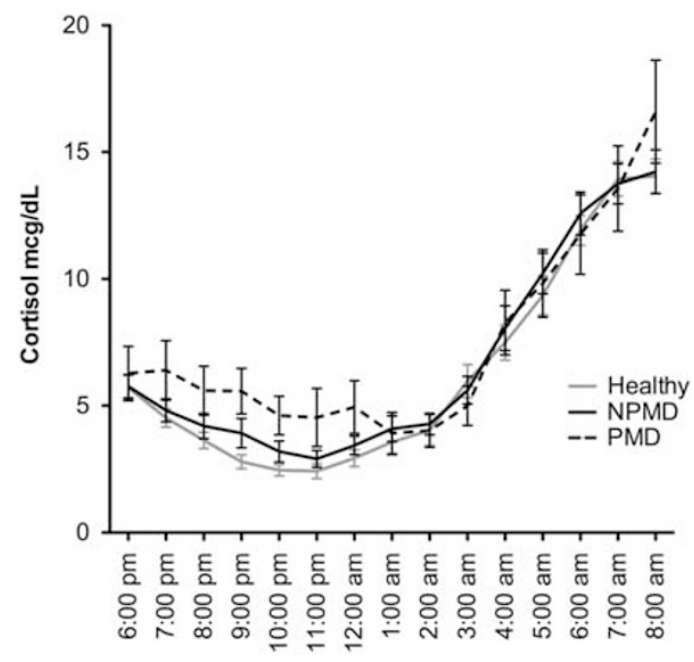

Figure 5 Cortisol secretion is elevated in the psychotic major depression group during the 1800-0100-hour window, which typically represents the circadian nadir.

regional rates of receptor downregulation in depression, leading to desynchronization of the regions.

A disruption in the communication or regulatory balance between the hypothalamus, subgenual cortex, and HPA axis activity could be resulting in downstream effects on symptoms. However, the experience of symptoms could also be driving connectivity and HPA axis changes. The data presented here suggest that disruptions in hypothalamic connectivity to the subgenual cortex and cortisol hypersecretion are both associated with overall increases in depressive symptoms but that cortisol may be more closely linked with symptom severity once the disease state is 
Table 4 All Backwards Stepwise Multivariate Regression Models Initially Contain Age, Gender, Cortisol, and Hypothalamic Connectivity with BA24, BA25, and BA32

\begin{tabular}{|c|c|c|c|c|c|}
\hline Dependant variable & Groups in model & Variable & Standardized beta & $T$-value & Significant effects \\
\hline \multirow[t]{4}{*}{ HAMD 21} & All participants & Age & 0.187 & 1.935 & 0.056 \\
\hline & & Cortisol & 0.213 & 2.227 & 0.028 \\
\hline & All depressed & Gender & -0.242 & -1.993 & 0.051 \\
\hline & & Cortisol & 0.295 & 2.43 & 0.018 \\
\hline & & Cortisol & 0.238 & 2.543 & 0.013 \\
\hline & & BA32 & -0.228 & -2.404 & 0.018 \\
\hline & All depressed & Gender & -0.288 & -2.546 & 0.014 \\
\hline & & Cortisol & 0.41 & 3.616 & 0.001 \\
\hline BPRS & All participants & Cortisol & 0.207 & 2.147 & 0.034 \\
\hline
\end{tabular}

Abbreviations: BPRS, Brief Psychiatric Ratings Scale; HAMD, Hamilton Depression Ratings Scale.

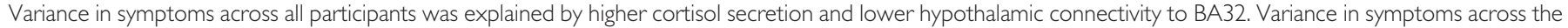
depressed groups, all of which had high levels of symptoms (see also Table I) was explained by higher cortisol secretion and gender.

established. Follow-up studies are needed to determine the causal relationships between hypothalamic connectivity, cortisol secretion/regulation, and symptom severity. Additional depression subtypes with HPA-axis dysregulation such as the melancholic subtype, may also have similar or even greater functional connectivity disruptions between the hypothalamus and the subgenual cortex.

This study had several limitations. First, it is difficult to recruit patients with PMD as it is a relatively rare depression subtype representing $18.5 \%$ of patients who meet the criteria major depression and $0.4 \%$ of the general population (Ohayon, 2002). Adding to this challenge is that psychotic symptoms tend to resolve quickly after treatment with antipsychotic medication. Therefore, it was not feasible to recruit PMD patients with active psychotic symptoms who were either drug free or even patients who were on standardized medication regimes. However, to limit confounding influences of switching/reducing psychiatric medication regimens and potential ethic concerns of having seriously ill medication-free patients, we required all participants to have stable medications for at least 1 week before participation. These medications could be impacting functional connectivity in unexpected ways; however, additional studies will be needed to amass a sample size of PMD patients that will provide the statistical power necessary to delineate the effects of specific medication on PMD functional connectivity patterns. Another limitation is that the PMD group had higher total symptoms scores on the HAM-D and the endogenomorphic subscale as compared with depressed patients without psychotic symptoms (Table 1). These differences arise from several items on the HAM-D that exploit core symptoms of psychosis, such as 'unusual thought content'. No significant connectivity or cortisol secretion differences were observed between NPMD and PMD regardless of if these total symptoms scores were included as a covariate or not.

\section{CONCLUSIONS}

This study provides the first descriptions of functional connectivity between the hypothalamus and subgenual cortex and also the first evidence that this relationship is perturbed in patients with PMD. The main a priori hypotheses in this study were only partially confirmed. Only some patients (PMD subtype) can be confirmed as having degraded hypothalamic resting-state functional connectivity with the subgenual cortex. PMD patients did present with the most degraded connectivity, greatest cortisol dysregulation, and the most severe symptoms. Both degraded connectivity and cortisol dysregulation were also associated with greater symptoms although cortisol dysregulation may be more associated with differences symptom differences amongst depressed patients, whereas degraded connectivity may be more associated with the symptom differences between healthy patients and depressed patients.

Longitudinal studies are required to address if these connectivity disruptions resolve alongside clinical improvements or predict relapse rates. Additional follow-up studies are needed to determine the hypothalamus-subgenual cortex-HPA axis circuit's potential contribution to the symptoms of depression.

\section{FUNDING AND DISCLOSURE}

Dr Reiss has received Consulting fee from Novartis Pharmaceuticals. Dr Schatzberg has served as a consultant to Bay City Capital, BrainCells, CeNeRx, Cervel, Depomed, Eli Lilly, EnVivo (Forum), Genentech, Gilead, Jazz, Lundbeck/Takeda, McKinsey, Merck*, MSI, Naurex, Neuronetics, Novadel, Pfizer ${ }^{\star}$, PharmaNeuroBoost* ${ }^{\star}$, Sunovion, Synosia, and Xhale. Dr Schatzberg has had equity in Amnestix, BrainCells, CeNeRx, Cervel, Corcept ${ }^{*}$ (co-founder), Delpor, Forest Labs, Merck*, Neurocrine, Novadel, Pfizer*, 
PharmaNeuroBoost, Seattle Genetics*, Somaxon, Synosia, Titan, and Xhale*. He is a named inventor on pharmacogenetic use patents on glucocorticoid antagonists and on prediction of antidepressant response. Dr Schatzberg has also received speaking fees from Merck. ${ }^{*}$ Greater than $\$ 10,000 /$ year in income or current equity value. The remaining authors declare no conflict of interest.

\section{REFERENCES}

Carroll BJ, Curtis GC, Davies BM, Mendels J, Sugerman AA (1976). Urinary free cortisol excretion in depression. Psychol Med 6: 43-50.

Damasio H (1995). Human Brain Anatomy in Computerized Images. Oxford University Press: New York.

Grecius M, Flores B, Menon V, Glover G, Solvason B, Kenna H et al (2007). Resting-state functional connectivity in major depression: abnormally increased contributions from subgenual cingulate cortex and thalamus. Biol Psychiatry 62: 429-437.

Greden JF, Gardner R, King D, Grunhaus L, Carroll BJ, Kronfol Z (1983). Dexamethasone suppression tests in antidepressant treatment of melancholia. The process of normalization and test-retest reproducibility. Arch Gen Psychiatry 40: 493-500.

Hamani C, Mayberg H, Stone S, Laxton A, Haber S, Lozano AM (2011). The subcallosal cingulate gyrus in the context of major depression. Biol Psychiatry 69: 301-308.

Hamilton M (1960). A rating scale for depression. J Neurol Neurosurg Psychiatry 23: 56-62.

Johansen-Berg H, Gutman DA, Behrens TEJ, Matthews PM, Rushworth MFS, Katz E et al (2008). Anatomical connectivity of the subgenual cingulate region targeted with deep brain stimulation for treatment-resistant depression. Cereb Cortex 18: 1374-1383.

Keller J, Flores B, Gomez RG, Solvason HB, Kenna H, Williams GH et al (2006). Cortisol circadian rhythm alterations in psychotic major depression. Biol Psychiatry 60: 275-281.

Lane RD, Weidenbacher H, Smith R, Fort C, Thayer JF, Allen JJB (2013). Subgenual anterior cingulate cortex activity covariation with cardiac vagal control is altered in depression. J Affect Disord 150: $565-570$.

Lembke A, Gomez R, Tenakoon L, Keller J, Cohen G, Williams GH et al (2012). The mineralocorticoid receptor agonist, fludrocortisone, differentially inhibits pituitary-adrenal activity in humans with psychotic major depression. Psychoneuroendocrinology 38: 115-121.

Lozano AM, Giacobbe P, Hamani C, Rizvi SJ, Kennedy SH, Kolivakis TT et al (2012). A multicenter pilot study of subcallosal cingulate area deep brain stimulation for treatmentresistant depression. J Neurosurg 116: 315-322.

Macey PM, Macey KE, Kumar R, Harper RM (2004). A method for removal of global effects from fMRI time series. Neuroimage 22: 360-366.

Mai JK, Assheuer J, Paxinos G1 (1997). Atlas of the Human Brain. Academic Press: San Diego, CA.

Mayberg HS, Brannan SK, Mahurin RK, Jerabek PA, Brickman JS, Tekell JL et al (1997). Cingulate function in depression: a potential predictor of treatment response. Neuroreport 8: 1057-1061.

Mayberg HS, Liotti M, Brannan SK, McGinnis S, Mahurin RK, Jerabek PA et al (1999). Reciprocal limbic-cortical function and negative mood: converging PET findings in depression and normal sadness. Am J Psychiatry 156: 675-682.
Nelson JC, Davis JM (1997). DST studies in psychotic depression: a meta-analysis. Am J Psychiatry 154: 1497-1503.

Ohayon MM (2002). Prevalence of depressive episodes with psychotic features in the general population. Am J Psychiatry 159: 1855-1861.

Ongür D, An X, Price JL (1998a). Prefrontal cortical projections to the hypothalamus in macaque monkeys. J Comp Neurol 401: 480-505.

Ongür D, Drevets WC, Price JL (1998b). Glial reduction in the subgenual prefrontal cortex in mood disorders. Proc Natl Acad Sci USA 95: 13290-13295.

Osuch EA, Ketter TA, Kimbrell TA, George MS, Benson BE, Willis MW et al (2000). Regional cerebral metabolism associated with anxiety symptoms in affective disorder patients. Biol Psychiatry 48: 1020-1023.

Patel PD, Lopez JF, Lyons DM, Burke S, Wallace M, Schatzberg AF (2000). Glucocorticoid and mineralocorticoid receptor mRNA expression in squirrel monkey brain. J Psychiatr Res 34: 383-392.

Phan KL, Wager T, Taylor SF, Liberzon I (2002). Functional neuroanatomy of emotion: a meta-analysis of emotion activation studies in PET and fMRI. Neuroimage 16: 331-348.

Rempel-Clower NL, Barbas H (1998). Topographic organization of connections between the hypothalamus and prefrontal cortex in the rhesus monkey. J Comp Neurol 398: 393-419.

Rhoades HM, Overall J (1988). The semistructured BPRS interview and rating guide. Psychopharmacol Bull 24: 101-104.

Stetler C, Miller GE (2011). Depression and hypothalamicpituitary-adrenal activation: a quantitative summary of four decades of research. Psychosom Med 73: 114-126.

Sudheimer K, Winn B, Garrett K, Shoaps J, Davies K, Fobbs A. The Human Brain Atlas, Michigan State University. Available at $<$ https://www.msu.edu/ brains/brains/human/index.html $>$.

Sudheimer KD, Abelson JL, Taylor SF, Martis B, Welsh RC, Warner C et al (2013). Exogenous glucocorticoids decrease subgenual cingulate activity evoked by sadness. Neuropsychopharmacology 38: 826-845.

Thase ME, Hersen M, Bellack AS, Himmelhoch JM, Kupfer DJ (1983). Validation of a Hamilton subscale for endogenomorphic depression. J Affect Disord 5: 267-278.

Tzourio-Mazoyer N, Landeau B, Papathanassiou D, Crivello F, Etard O, Delcroix N et al (2002). Automated anatomical labeling of activations in SPM using a macroscopic anatomical parcellation of the MNI MRI single-subject brain. Neuroimage 15: 273-289.

Veazey RB, Amaral DG, Cowan WM (1982). The morphology and connections of the posterior hypothalamus in the cynomolgus monkey (Macaca fascicularis). II. Efferent connections. J Comp Neurol 207: 135-156.

Watzka M, Bidlingmaier F, Beyenburg S, Henke RT, Clusmann H, Elger CE et al (2000). Corticosteroid receptor mRNA expression in the brains of patients with epilepsy. Steroids 65: 895-901.

Webster MJ, Knable MB, O'Grady J, Orthmann J, Weickert CS (2002). Regional specificity of brain glucocorticoid receptor mRNA alterations in subjects with schizophrenia and mood disorders. Mol Psychiatry 7: 985-994924.

Zobel AW, Nickel T, Sonntag A, Uhr M, Holsboer F, Ising M (2001). Cortisol response in the combined dexamethasone/CRH test as predictor of relapse in patients with remitted depression. a prospective study. J Psychiatr Res 35: 83-94.

Zobel AW, Yassouridis A, Frieboes RM, Holsboer F (1999). Prediction of medium-term outcome by cortisol response to the combined dexamethasone-CRH test in patients with remitted depression. Am J Psychiatry 156: 949-951. 